\title{
Analysis of OFDM Based Bidirectional Relay Network with Multiple Antennas in the Presence of Phase Noise
}

Senthil Kumaran V.N ( $\sim$ vnsenthilkumaranece@gmail.com )

VSB Engineering College

Andrew Roobert A

Francis Xavier Engineering College

\section{Research Article}

Keywords: Bidirectional Relay Network, Orthogonal Frequency Division Multiplexing (OFDM), Signal-toInterference Noise Ratio (SINR), Phase Noise

Posted Date: March 16th, 2021

DOI: https://doi.org/10.21203/rs.3.rs-261179/v1

License: (c) This work is licensed under a Creative Commons Attribution 4.0 International License.

Read Full License 


\title{
Analysis of OFDM Based Bidirectional Relay Network with Multiple Antennas in the Presence of Phase Noise
}

\author{
V.N.Senthil Kumaran ${ }^{1}$, A.Andrew Roobert ${ }^{2}$ \\ Department of Electronics and Communication Engineering, \\ ${ }^{1}$ VSB Engineering College, Karur - 639 111, Tamilnadu, India \\ ${ }^{2}$ Francis Xavier Engineering College, Tirunelveli - 627 003, Tamilnadu, India \\ vnskchennai@gmail.com ${ }^{1}$, andrewroobert@gmail.com²
}

\begin{abstract}
:
In this article, a bidirectional relay network based on OFDM with multiple antennas at source nodes is proposed. The concepts of Transmit Antenna Selection (TAS) and Maximal Ratio Combining (MRC) are applied at the source nodes in first and second time slots respectively to exploit spatial diversity to improve the outage performance in the presence of phase noise. Modeling and analysis of the proposed bidirectional relay network in the presence of phase noise are briefly discussed. Analytical results have been verified by Monte-Carlo simulations. The performance of the proposed work is compared with the literature, and better performance is observed in the proposed work.
\end{abstract}

\section{KEYWORDS:}

Bidirectional Relay Network, Orthogonal Frequency Division Multiplexing (OFDM), Signalto-Interference Noise Ratio (SINR), Phase Noise

\section{$1 \quad$ INTRODUCTION}

The proposed transceiver employs multiple antennas at the source nodes and transmit antenna is selected such that the link between the source node and relay provides high SINR at each subcarrier level. The transmit antenna selection information is sent through a robust low data rate binary symmetric channel to the relay node. Maximal ratio combining is done at the source nodes in second time slot. Orthogonal Frequency Division Multiplexing (OFDM) system transmits numerous high data streams through narrow band flat fading subchannels to achieve high spectral efficiency over wide band channels [1]. OFDM exhibits a significant sensitivity to the phase noise of the oscillator used for frequency down-conversion at the portable receiver [2]. For this reason, it is important to evaluate the 
impact of the phase noise on the system performance. This helps in understanding the meaning of small phase noise and how it reflects on the proper parameters selection of a specific OFDM system. Further, phase noise must be carefully considered when designing an OFDM-based communication system since an accurate prediction of the tolerable phase noise can allow the system and RF engineers to relax specifications.

Though much work has been done on the performance analysis of PLNC wireless relay network in the literature, in most of the works, it is assumed that both carrier and phase are perfectly synchronized between the transmitter and at the receiver. It is customary to characterize the achievable performance of the OFDM based PLNC relay network due to practical constraints such as I/Q imbalance and phase noise. The first objective of this paper is to model the proposed transceiver for OFDM based bidirectional relay network in the presence of phase noise. The second objective is to analyze the effect of phase noise in the outage performance at relay node in first time slot and source nodes in second time slot. The third objective is to study the overll end to end outage performance of the propsoed OFDM based bidirectional relay network in the presence of phase noise. The organization of this article is as follows. Section 2 describes the system model. Signal-to-Interference Noise Ratio (SINR) is analysed in section 3. Outage analysis is performed in section 4. The results and discussion are detailed in section 5. This article is concluded in section 6.

\section{SYSTEM MODEL}

Consider a bidirectional relay network consisting of two source nodes $S_{1}, S_{2}$ with $M$ antennas and a relay node $R$ with a single antenna shown in Figure 1. The bidirectional relay network operates in a half duplex mode [3-6]. The channels between $S_{1}$ to $R$ and $S_{2}$ to $R$ are assumed to be quasi-static frequency selective fading such that the channel statistics does not change within a transmission duration of one frame but can vary from one frame to another.

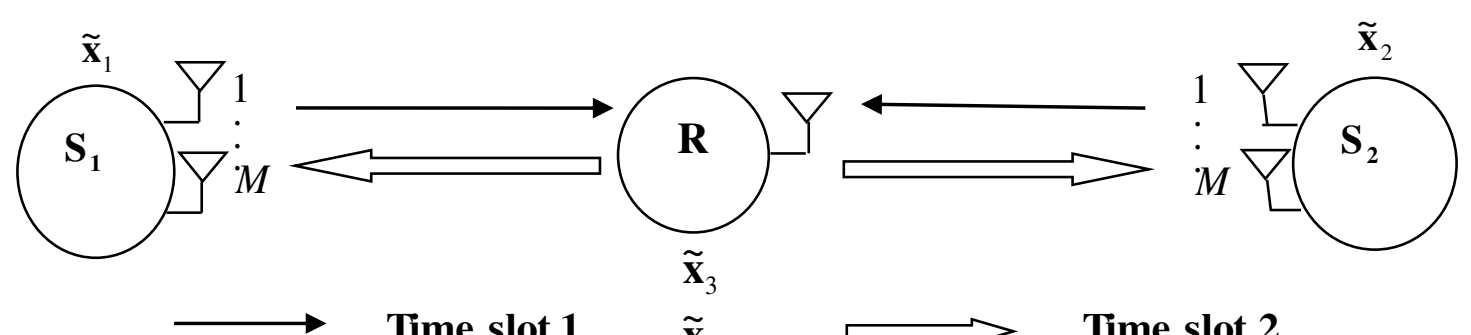

$\longrightarrow$ Time slot $1 \quad \tilde{\mathbf{x}}_{3} \longrightarrow$ Time slot 2 


\section{Figure 1 System model}

The baseband equivalent channel impulse response between the $b^{\text {th }}$ transmit antenna of source node $S_{m}$ and relay node $R$ is denoted by $\mathbf{g}_{m, b}=\left[g_{m, b}(0), \ldots g_{m, b}\left(L_{m, b}-1\right)\right]^{T}, m=1,2, b=1,2, \ldots M . \quad$ The elements of $\mathbf{g}_{m, b}$ are independent and identically distributed complex Gaussian with zero mean and unit variance. $L_{m, b}$ represents the number of taps of the channels between $b^{\text {th }}$ antenna of source node $S_{m}$ and the relay node $R$. The frequency response of $\mathbf{g}_{m, b}$ is defined as

$$
\tilde{g}_{m, b}[k]=\frac{1}{\sqrt{N}} \sum_{l=0}^{L_{m, b}-1} g_{m, b}(l) \exp \left(\frac{-j 2 \pi k l}{N}\right), k=0,1,2, \ldots . . N-1, m=1,2, b=1,2, \ldots M
$$

where $N$ is the number of subcarriers in OFDM symbol. At each subcarrier, one of the $M$ transmit antennas is selected for transmission at source nodes $S_{1}$ and $S_{2}$. Let $m_{1, k}$ and $m_{2, k}$ represent the selected antenna at $k^{\text {th }}$ subcarrier in source nodes $S_{1}$ and $S_{2}$. The criterions for the selection of transmit antenna at $k^{\text {th }}$ subcarrier in source nodes $S_{1}$ and $S_{2}$ are defined as

$$
\begin{aligned}
& a_{1, k}=\underset{b \in(1,2 . M)}{\arg \max }\left|\tilde{g}_{1, b}[k]\right|^{2}, k=0,1 \ldots N-1 \\
& a_{2, k}=\underset{b \in(1,2 \ldots M)}{\arg \max }\left|\tilde{g}_{2, b}[k]\right|^{2}, k=0,1 \ldots N-1
\end{aligned}
$$

The antenna selection information is transmitted to the relay node $R$ through a robust binary symmetric channel. During time slot 1 , both the source nodes $S_{1}$ and $S_{2}$ transmit OFDM signals with $N$ information symbols [7]. The Cyclic Prefix (CP) of length $L_{C P}$, to the relay node $R$. The $\mathrm{CP}$ length, $L_{C P}$ satisfies the condition that $L_{C P} \geq \max \left\{L_{m, b}-1, m=1,2, b=1, \ldots . M\right\}$ to avoid Inter-Symbol Interference (ISI). In the presence of phase noise, the received signal at the relay node $R$ after taking DFT, is given by

$$
\begin{aligned}
\tilde{y}_{r}[k] & =\left[\sqrt{E_{1}} \tilde{g}_{1, a_{1, k}}[k] \tilde{x}_{1}[k] \tilde{p}_{1 r}[0]+\sqrt{E_{2}} \tilde{g}_{2, a_{2, k}}[k] \tilde{x}_{2}[k] \tilde{p}_{2 r}[0]\right] \\
+ & {\left[\sum_{q=0, q \neq k}^{N-1}\left(\sqrt{E_{1}} \tilde{g}_{1, a_{1, q}}[q] \tilde{x}_{1}[q] \tilde{p}_{1 r}[k-q]+\sqrt{E_{2}} \tilde{g}_{2, a_{2, q}}[q] \tilde{x}_{2}[q] \tilde{p}_{2 r}[k-q]\right)\right]+\tilde{n}_{r}[k] } \\
k & =0,1, \ldots N-1
\end{aligned}
$$


where $E_{1}$ and $E_{2}$ are the transmission powers at source nodes $S_{1}$ and $S_{2}$ respectively [8]. $\tilde{n}_{r}[k]$ is zero mean circularly symmetric complex Gaussian noise with variance $\sigma_{r}^{2}, \tilde{p}_{m r}[0]$ is the Common Phase Error (CPE) and $\tilde{p}_{m r}[k-q]$ is the Inter-Carrier Interference (ICI) due to the phase noise [9]. Equation (4) is rewritten simply as

$$
\begin{aligned}
\tilde{y}_{r}[k] & =\sum_{m=1}^{2} \sqrt{E_{m}} \tilde{g}_{m, a_{m, k}}[k] \tilde{x}_{m}[k] \tilde{p}_{m r}[0]+\sum_{m=1}^{2} \sum_{q=0, q \neq k}^{N-1}\left(\sqrt{E_{m}} \tilde{g}_{m, a_{m . k}}[k] \tilde{x}_{m}[q] \tilde{p}_{m r}[k-q]\right)+\tilde{n}_{r}[k] \\
k & =0,1, \ldots N-1
\end{aligned}
$$

The transmitted symbol sequences $\tilde{\mathbf{x}}_{1}$ and $\tilde{\mathbf{x}}_{2}$ are jointly estimated using Maximum Likelihood (ML) principle at relay node $R$. The estimate of the $k^{\text {th }}$ symbols of both the sequence is given by

$$
\begin{array}{r}
\left\{\hat{x}_{1}[k], \hat{x}_{2}[k]\right\}=\underset{\left(\tilde{x}_{1}[k], \tilde{x}_{2}[k]\right)\left\{\{-1,1\}^{2}\right.}{\arg \min }\left|\tilde{y}_{r}[k]-\sqrt{E_{1}} \tilde{g}_{1, a_{1, k}}[k] \tilde{x}_{1}[k]-\sqrt{E_{2}} \tilde{g}_{2, a_{2, k}}[k] \tilde{x}_{2}[k]\right|^{2} \\
k=0,1, \ldots, N-1
\end{array}
$$

where $\{-1,1\}^{2}$ is the second order extension of the set $\{1,-1\}$. Using the joint estimation of the symbols of $\tilde{\mathbf{x}}_{1}$ and $\tilde{\mathbf{x}}_{2}$ a new sequence $\tilde{\mathbf{x}}_{r}$ is formed as $\tilde{x}_{r}[k]=\hat{x}_{1}[k] \hat{x}_{2}[k], k=0,1, \ldots, N-1$ at the relay node $R$. Then, an OFDM signal corresponding to the signal vector $\tilde{x}_{r}[k]$ is generated by taking IDFT and appending CP of length, $L_{C P}$.

During the time slot II, the relay node $R$ broadcasts the OFDM signal to the source nodes $S_{1}$ and $S_{2}$. Assuming perfect timing and frequency offset, the received signals at $b^{\text {th }}$ receive antenna of the source nodes $S_{1}$ and $S_{2}$ perturbed by phase noise are given by

$$
\begin{gathered}
\tilde{y}_{1, b}[k]=\sqrt{E_{r}} \tilde{g}_{1, b}[k] \tilde{x}_{r}[k] \tilde{p}_{r 1}[0]+\sum_{q=0, q \neq k}^{N-1} \sqrt{E_{r}} \tilde{g}_{1, b}[q] \tilde{x}_{r}[q] \tilde{p}_{r 1}[k-q]+\tilde{n}_{1, b}[k] \\
k=0,1, \ldots, N-1, b=1, \ldots M
\end{gathered}
$$




$$
\begin{gathered}
\tilde{y}_{2, b}[k]=\sqrt{E_{r}} \tilde{g}_{2, b}[k] \tilde{x}_{r}[k] \tilde{p}_{r 2}[0]+\sum_{q=0, q \neq k}^{N-1} \sqrt{E_{r}} \tilde{g}_{2, b}[q] \tilde{x}_{r}[q] \tilde{p}_{r 2}[k-q]+\tilde{n}_{2, b}[k] \\
k=0,1, \ldots, N-1, b=1, \ldots M
\end{gathered}
$$

Assuming that the channel frequency response vectors are known at source nodes $S_{1}$ and $S_{2}$, the Maximal Ratio Combiner (MRC) output at nodes $S_{1}$ and $S_{2}$ are given by

$$
\begin{aligned}
& \tilde{y}_{1}[k]=\sum_{b=1}^{M}\left[\sqrt{E_{r}}\left|\tilde{g}_{1, b}[k]\right|^{2} \tilde{x}_{r}[k]\right] \tilde{p}_{r 1}[0]+\sum_{b=1}^{M} \sum_{q=0, q \neq k}^{N-1} \sqrt{E_{r}} \tilde{g}_{1, b}[q] \tilde{g}_{1, b}^{*}[k] \tilde{x}_{r}[q] \tilde{p}_{r 1}[k-q] \\
& +\sum_{b=1}^{M} \tilde{n}_{1, b}[k] \tilde{g}_{1, b}^{*}[k], k=0,1, \ldots, N-1
\end{aligned}
$$

$$
\begin{aligned}
& \tilde{y}_{2}[k]=\sum_{b=1}^{M}\left[\sqrt{E_{r}}\left|\tilde{g}_{2, b}[k]\right|^{2} \tilde{x}_{r}[k]\right] \tilde{p}_{r 2}[0]+\sum_{b=1}^{M} \sum_{q=0, q \neq k}^{N-1} \sqrt{E_{r}} \tilde{g}_{2, b}[q] \tilde{g}_{2, b}^{*}[k] \tilde{x}_{r}[q] \tilde{p}_{r 2}[k-q] \\
& +\sum_{b=1}^{M} \tilde{n}_{2, b}[k] \tilde{g}_{2, b}^{*}[k], k=0,1, \ldots, N-1
\end{aligned}
$$

where $E_{r}$ is the transmission power at relay node $R$ and $\tilde{p}_{r, 1}[k-q], \tilde{p}_{r, 2}[k-q]$ are the ICI due to the phase noise. $\tilde{n}_{1, b}[k]$ and $\tilde{n}_{2, b}[k]$ are circularly symmetric complex Gaussian noises with zero mean and variances $\sigma_{n_{1}}^{2}$ and $\sigma_{n_{2}}^{2}$ respectively [10-12]. The ML estimate of $\hat{x}_{r}^{(1)}[k]$ and $\hat{x}_{r}^{(2)}[k]$ at $S_{1}$ and $S_{2}$ respectively, are given by

$$
\begin{aligned}
& \hat{x}_{r}^{(1)}[k]=\underset{\tilde{x}_{r}[k] \in\{-1,1\}}{\arg \min }\left|\tilde{y}_{1}[k]-\sum_{b=1}^{M}\left[\sqrt{E_{r}}\left|\tilde{g}_{1, b}[k]\right|^{2} \tilde{x}_{r}[k]\right]\right|^{2} k=0,1, \ldots, N-1 \\
& \hat{x}_{r}^{(2)}[k]=\underset{\tilde{x}_{r}[k] \in\{-1,1\}}{\arg \min }\left|\tilde{y}_{2}[k]-\sum_{b=1}^{M}\left[\sqrt{E_{r}}\left|\tilde{g}_{2, b}[k]\right|^{2} \tilde{x}_{r}[k]\right]\right|^{2} k=0,1, \ldots, N-1
\end{aligned}
$$

Then, $S_{1}$ estimates $\hat{x}_{2}[k]$ by multiplying the symbol $\hat{x}_{r}^{(1)}[k]$ with its own symbol $\tilde{x}_{1}[k]$ and $S_{2}$ estimates $\hat{x}_{1}[k]$ by multiplying the symbol $\hat{x}_{r}^{(2)}[k]$ with its own symbol $\tilde{x}_{2}[k]$ as follows: 


$$
\begin{array}{ll}
\hat{x}_{2}[k]=\hat{x}_{r}^{(1)}[k] \tilde{x}_{1}[k] & k=0,1, \ldots, N-1 \\
\hat{x}_{1}[k]=\hat{x}_{r}^{(2)}[k] \tilde{x}_{2}[k] & k=0,1, \ldots, N-1
\end{array}
$$

\section{SINR ANALYSIS}

The Signal-to-Interference Noise Ratio (SINR) expressions at the nodes of the bidirectional relay network in the first and second time slots are derived in the presence of phase noise and multiple antennas. It is assumed that the CPE term has been compensated.

\subsection{SINR Analysis at Time Slot - I}

It is assumed that the channel frequency response vectors $\tilde{\mathbf{g}}_{m, a_{1}}$ and $\tilde{\mathbf{g}}_{m, a_{2}}, m=1,2$ are known at the relay node $R$. Using the Equation (5.5) instantaneous SINR for the signals from $S_{1}$ and $S_{2}$ to the relay node $R$ at the $k^{\text {th }}$ subcarrier, during time slot-I conditioned on the individual channel knowledge, are expressed as

$$
\begin{aligned}
& \gamma_{1, \text { relay }}^{P-P N}[k]=\frac{E\left[E_{1}\left|\tilde{x}_{1}[k]\right|^{2}\right]\left|\tilde{g}_{1, a_{1, k}}[k]\right|^{2}\left|\tilde{p}_{1 r}[0]\right|^{2}}{E\left[\left|\sum_{q=0, q \neq k}^{N-1}\left(\sqrt{E_{1}} \tilde{x}_{1}[q] \tilde{g}_{1, a_{1, k}}[k] \tilde{p}_{1 r}[k-q]\right)\right|^{2}\right]+E\left[\left|\tilde{n}_{r}[k]\right|^{2}\right]} \\
& \gamma_{2, \text { relay }}^{P-P N}[k]=\frac{E\left[E_{2}\left|\tilde{x}_{2}[k]\right|^{2}\right]\left|\tilde{g}_{2, a_{2, k}}[k]\right|^{2}\left|\tilde{p}_{2 r}[0]\right|^{2}}{E\left[\mid \sum_{q=0, q \neq k}^{N-1}\left(\left.\sqrt{E_{2}} \tilde{x}_{2}[q] \tilde{g}_{2, a_{2, k}}[k] \tilde{p}_{2 r}[k-q]\right|^{2}\right]+E\left[\left|\tilde{n}_{r}[k]\right|^{2}\right]\right.}
\end{aligned}
$$

In general, Equation (15) and Equation (16) can be combined as

$$
\gamma_{m, \text { relay }}^{P-P N}[k]=\frac{E\left[E_{m}\left|\tilde{x}_{m}[k]\right|^{2}\right]\left|\tilde{g}_{m, a_{m, k}}[k]\right|^{2}\left|\tilde{p}_{m r}[0]\right|^{2}}{E\left[\mid \sum_{q=0, q \neq k}^{N-1}\left(\left.\sqrt{E_{m}} \tilde{x}_{m}[q] \tilde{g}_{m, a_{m, k}}[k] \tilde{p}_{m r}[k-q]\right|^{2}\right]+E\left[\left|\tilde{n}_{r}[k]\right|^{2}\right]\right.}, m=1,2
$$


Using Cauchy's Schwartz inequality, the denominator term can be written as:

$$
\begin{aligned}
& E\left[\left.|| \sum_{q=0, q \neq k}^{N-1} \sqrt{E_{m}} \tilde{x}_{m}[q] \tilde{g}_{m, a_{m, k}}[k] \tilde{p}_{m r}[k-q]\right|^{2}\right] \leq E\left[\sum_{q=0, q \neq k}^{N-1} E_{m}\left|\tilde{x}_{m}[q]\right|^{2}\right] \\
& \sum_{q=0, q \neq k}^{N-1}\left|\tilde{p}_{m r}[k-q]\right|_{q=0, q \neq k}^{2-1}\left|\tilde{g}_{m, a_{m, k}}[q]\right|^{2}
\end{aligned}
$$

Since $E\left[\left|\tilde{x}_{m}[k]\right|^{2}\right]=1$ and $E\left[\sum_{\substack{q=0 \\ q \neq k}}^{N-1}\left|\tilde{x}_{m}(q)\right|^{2}\right]=1$, the expression for SINR in

Equation (18) is expressed as

$$
\gamma_{m, \text { relay }}^{P-P N}[k] \geq \frac{E_{m}\left|\tilde{g}_{m, a_{m, k}}[k]\right|^{2}\left|\tilde{p}_{m r}(0)\right|^{2}}{E_{m} \sum_{q=0, q \neq k}^{N-1}\left|\tilde{p}_{m r}[k-q]^{2}\right| \sum_{q=0, q \neq k}^{N-1}\left|\tilde{g}_{m, a_{m, k}}[q]\right|^{2}+\sigma_{r}^{2}}, \quad m=1,2
$$

Assuming that the CPE term $\tilde{p}_{m r}(0)$ is compensated, Equation (19) can be further simplified as

$$
\gamma_{m, \text { relay }}^{P-P N}[k] \geq \frac{\left|\tilde{g}_{m, a_{m . k}}[k]\right|^{2}}{\sum_{q=0, q \neq k}^{N-1}\left|\tilde{p}_{m r}[k-q]\right| \sum_{q=0, q \neq k}^{N-1}\left|\tilde{g}_{m, a_{m, k}}[q]\right|^{2}+\frac{\sigma_{r}^{2}}{E_{m}}}, \quad m=1,2
$$

The variance of $\tilde{p}_{m r}(0)$ is determined as $\sigma_{p}^{2}=1-\frac{\pi\left(\beta_{m}+\beta_{r}\right) N}{3 R_{d}}$. Therefore $\sum_{q=0, q \neq k}^{N-1}\left|\tilde{p}_{m r}[k-q]\right|^{2}=\frac{\pi\left(\beta_{m}+\beta_{r}\right) N}{3 R_{d}}$. Substituting this value in Equation (20) $\gamma_{m, \text { relay }}^{P-P N}[k]$ is written as

$$
\gamma_{m, \text { relay }}^{P-P N}[k] \geq \frac{\left|\tilde{g}_{m, a_{m, k}}[k]\right|^{2}}{\frac{\pi\left(\beta_{m}+\beta_{r}\right) N T_{s}}{3} \sum_{q=0, q \neq k}^{N-1}\left|\tilde{g}_{m, a_{m, k}}[q]\right|^{2}+\frac{\sigma_{r}^{2}}{E_{m}}}, \quad m=1,2
$$


During the time slot II, the source node $S_{m}$ experiences receive diversity. Maximal ratio combining (MRC) is performed at the source node $S_{m}$. Using the Equation (9), the instantaneous SINR for the signals from the relay node $R$ to the destination node $S_{1}$ at $k^{\text {th }}$ subcarrier, during the time slot II is written as

$$
\gamma_{1, d e s t-M R C}^{P P N}[k]=\frac{E\left|\sum_{b=1}^{M}\left[\sqrt{E_{r}}\left|\tilde{g}_{1, b}[k]\right|^{2} \tilde{x}_{r}[k]\right] \tilde{p}_{r 1}[0]\right|^{2}}{E\left|\sum_{b=1}^{M} \sum_{q=0, q \neq k}^{N-1} \sqrt{E_{r}} \tilde{g}_{1, b}[q] \tilde{g}_{1, b}^{*}[k] \tilde{x}_{r}[q] \tilde{p}_{r 1}[k-q]\right|^{2}+E\left|\sum_{b=1}^{M} \tilde{n}_{1, b}[k] \tilde{g}_{1, b}^{*}[k]\right|^{2}}, k=0,1, \ldots, N-1
$$

Using Cauchy - Schwartz inequality the denominator term is simplified as

$$
\begin{aligned}
E\left|\sum_{b=1}^{M} \sum_{q=0, q \neq k}^{N-1} \sqrt{E_{r}} \tilde{g}_{1, b}[q] \tilde{g}_{1, b}^{*}[k] \tilde{x}_{r}[q] \tilde{p}_{r 1}[k-q]\right|^{2} \\
\quad \leq E\left[E_{r} \sum_{q=0, q \neq k}^{N-1}\left|\tilde{x}_{r}[q]\right|^{2}\right] \sum_{q=0, q \neq k}^{N-1}\left|\tilde{p}_{r 1}[k-q]\right|^{2} \sum_{b=1}^{M}\left|\tilde{g}_{1, b}[k]\right|^{2} \sum_{b=1}^{M} \sum_{q=0, q \neq k}^{N-1}\left|\tilde{g}_{1, b}[q]\right|^{2}
\end{aligned}
$$

Substituting Equation (23), the SINR expression is written as

$$
\gamma_{1, \text { dest }-M R C}^{P-P N}[k] \geq \frac{E\left|\sum_{b=1}^{M}\left[\sqrt{E_{r}} \tilde{x}_{r}[k]\right] \tilde{p}_{r 1}[0]\right|^{2}\left(\left.\left.\left|\sum_{b=1}^{M}\right| \tilde{g}_{1, b}[k]\right|^{2}\right|^{2}\right)}{\left(E\left[E \sum_{r}^{N-1}\left|\tilde{x}_{q=0, q \neq k}[q]\right|^{2}\right] \sum_{q=0, q \neq k}^{N-1}\left|\tilde{p}_{r 1}[k-q]\right|^{2} \sum_{b=1}^{M}\left|\tilde{g}_{1, b}[k]\right|^{2} \sum_{b=1}^{M} \sum_{q=0, q \neq k}^{N-1}\left|\tilde{g}_{1, b}[q]\right|^{2}\right)}, k=0,1, \ldots, N-1
$$

Since $E\left[\left|\tilde{x}_{r}[k]\right|^{2}\right]=1, E\left[\sum_{\substack{q=0 \\ q \neq k}}^{N-1}\left|x_{r}(q)\right|^{2}\right]=1$ and assuming that the CPE term $\tilde{p}_{r 1}[0]$ in Equation (24) has been compensated, the SINR expression can be further simplified as 


$$
\gamma_{1, \text { dest }-M R C}^{P-P N}[k]=\frac{\left(\left.\left.\left|\sum_{b=1}^{M}\right| \tilde{g}_{1, b}[k]\right|^{2}\right|^{2}\right)}{\left(\left(\frac{\pi\left(\beta_{r}+\beta_{1}\right) N T_{s}}{3}\right)\left\{\sum_{b=1}^{M}\left|\tilde{g}_{1, b}[k]\right|^{2} \sum_{b=1}^{M} \sum_{q=0, q \neq k}^{N-1}\left|\tilde{g}_{1, b}[q]\right|^{2}\right\}+\frac{\sigma_{1}^{2}\left(\sum_{b=1}^{M}\left|\tilde{g}_{1, b}[k]\right|^{2}\right)}{E_{r}}\right)}
$$

Equation (25) is further simplified as

$$
\gamma_{1, \text { dest }-M R C}^{P-P N}[k]=\frac{\left(\sum_{b=1}^{M}\left|\tilde{g}_{1, b}[k]\right|^{2}\right)}{\left(\left(\frac{\pi\left(\beta_{r}+\beta_{1}\right) N T_{s}}{3}\right)\left\{\sum_{b=1}^{M} \sum_{q=0, q \neq k}^{N-1}\left|\tilde{g}_{1, b}[q]\right|^{2}\right\}+\frac{\sigma_{1}^{2}}{E_{r}}\right)}, k=0,1, \ldots, N-1
$$

Similarly, the instantaneous SINR expression at the source node $S_{2}$ during time slot II can be expressed as

$$
\gamma_{2, \text { dest }-M R C}^{P-P N}[k]=\frac{\left(\sum_{b=1}^{M}\left|\tilde{g}_{2, b}[k]\right|^{2}\right)}{\left(\left(\frac{\pi\left(\beta_{r}+\beta_{2}\right) N T_{s}}{3}\right)\left\{\sum_{b=1}^{M} \sum_{q=0, q \neq k}^{N-1}\left|\tilde{g}_{2, b}[q]\right|^{2}\right\}+\frac{\sigma_{2}^{2}}{E_{r}}\right)}, k=0,1, \ldots, N-1
$$

The generalized form of the SINR expression at time slot II with MRC at the source node $S_{m}$ is given by $\gamma_{m, \text { dest }-M R C}^{P-P N}[k]=\frac{\left(\sum_{b=1}^{M}\left|\tilde{g}_{m, b}[k]\right|^{2}\right)}{\left(\left(\frac{\pi\left(\beta_{r}+\beta_{m}\right) N T_{s}}{3}\right)\left\{\sum_{b=1}^{M} \sum_{q=0, q \neq k}^{N-1}\left|\tilde{g}_{m, b}[q]\right|^{2}\right\}+\frac{\sigma_{m}^{2}}{E_{r}}\right)}, k=0,1, \ldots, N-1, m=1,2$

\section{OUTAGE ANALYSIS}


In this section the effect of phase noise on the outage performance at the relay node $R$ and destination node $S_{m}$ are characterized for the proposed system. Let $P_{o u t, \gamma_{m}}^{P-P N}[\gamma]$ be the outage probability at the link between the source node $S_{m}$ and relay node $R$ at $k^{\text {th }}$ subcarrier in time slot I.

It is defined as $P_{\text {out }, \gamma_{m}}^{P-P N}[\gamma]=\operatorname{Pr}\left[\gamma_{m, \text { relay }}^{P-P N}[k] \leq \gamma_{T A S}\right]$.

where $\gamma_{T A S}=\max \left\{\gamma_{1}[k], \gamma_{2}[k], \ldots \gamma_{M}[k]\right\}$ is the maximum SINR of the $M$ transmit signals using TAS at the time slot I.

\subsection{Outage Analysis at Time Slot - I}

The upper bound outage probability at the relay node $R$ in time slot I with selection diversity at source nodes $S_{1}$ and $S_{2}$ during transmission is given by

$$
P_{\text {out }, \text { relay }}^{P-P(\text { Upper })}[\gamma]=\sum_{m=1}^{2} P_{\text {out }, \gamma_{m}}^{P-P N}[\gamma]
$$

$P_{o u t, \gamma_{m}}^{P-P N}[\gamma]$ represents the outage probability at the link between the source node $S_{m}$ and the relay node $R$ in $k^{\text {th }}$ subcarrier with selection diversity during transmission. Substituting for $\gamma_{m, \text { relay }}^{P-P N}[k]$ from Equation (21), it is written as

$$
P_{\text {out }, \text { relay }}^{P-P N(\text { Upper })}[\gamma]=\sum_{m=1}^{2} \operatorname{Pr}\left[\frac{\left|\tilde{g}_{m, a_{m, k}}[k]\right|^{2}}{\frac{\pi\left(\beta_{m}+\beta_{r}\right) N T_{s}}{3} \sum_{q=0, q \neq k}^{N-1}\left|\tilde{g}_{m, a_{m, k}}[q]\right|^{2}+\frac{\sigma_{r}^{2}}{E_{m}}} \leq \gamma_{T A S}\right]
$$

Let $\quad X_{m}=\left|\tilde{g}_{m, a_{m, k}}[k]\right|^{2}, \quad Y_{m}=\sum_{q=0, q \neq k}^{N-1}\left|\tilde{g}_{m, a_{m . k}}[q]\right|^{2}, \quad U_{m}=\frac{\pi\left(\beta_{m}+\beta_{r}\right) N T_{s}}{3} \quad$ and $V_{m}=\frac{\sigma_{r}^{2}}{E_{m}}=\frac{1}{S N R}$

Then, Equation (30) can be rewritten as

$$
P_{\text {out }, \text { relay }}^{P-P N(\text { Upper })}[\gamma]=\sum_{m=1}^{2} \operatorname{Pr}\left[\frac{X_{m}}{U_{m} Y_{m}+V_{m}} \leq \gamma_{T A S}\right]
$$


Using the PDF of $X_{m}$ and $Y_{m}$, Equation (31) can be written as

$$
P_{\text {out }, \text { relay }}^{P-P N \text { (Upper })}[\gamma]=\sum_{m=1}^{2}\left[\int_{0}^{\infty} \int_{0}^{\gamma\left(U_{m} y_{m}+V_{m}\right)} f_{X_{m}}\left(x_{m}\right) f_{Y_{m}}\left(y_{m}\right) d x_{m} d y_{m}\right]
$$

Since $\tilde{g}_{m, a_{m . k}}[k]$ is circularly symmetric complex Gaussian random variable with zero mean and unit variance, the random variable $X_{m}$ is chi - square distributed with 2 degrees of freedom and the random variable $Y_{m}$ is chi-square distributed with $2(N-1)$ degrees of freedom. The PDF of $X_{m}$ and $Y_{m}$ are expressed as

$$
\begin{aligned}
& f_{X_{m}}\left(x_{m}\right)=\exp \left(-x_{m}\right), \quad m=1,2 \\
& f_{Y_{m}}\left(y_{m}\right)=\frac{1}{\Gamma(N-1) !\left(\frac{1}{N-1}\right)^{N-1}} y^{N-2} \exp \left(\frac{-y}{1 /(N-1)}\right), \quad m=1,2
\end{aligned}
$$

Even though a single active antenna is selected at the source node, it is still possible to achieve $M$ diversity order in the source to relay link. Now substituting Equation (33) and Equation (34) in Equation (32) and evaluating the integrals, the outage probability $P_{o u t, \gamma_{m}}^{P-P N}[\gamma]$ at relay node $R$ during the time slot $\mathrm{I}$ is determined as

$$
P_{o u t, \gamma_{m}}^{P-P N}[\gamma]=\left[1-\exp \left(-\gamma_{T A S}\left\{\frac{1}{S N R}\left(1+\frac{\pi\left(\beta_{m}+\beta_{r}\right) N T_{s}}{3} \gamma_{T A S}\right)^{-(N-2)}\right\}\right)\right]^{M}, \quad m=1,2, M=2
$$

Substituting Equation (35) in (29), the upper bound outage probability at the relay node $R$ is determined as

$$
\begin{aligned}
& P_{\text {out }, \text { relay }}^{P-P N(\text { Upper })}[\gamma]=2-\exp \left[-\gamma_{\text {TAS }}\left\{\frac{1}{S N R}\left(1+\frac{\pi\left(\beta_{1}+\beta_{r}\right) \beta_{1} N T_{s}}{3} \gamma_{T A S}\right)^{-(N-2)}\right\}\right]^{M} \\
&-\exp \left[-\gamma_{T A S}\left\{\frac{1}{S N R}\left(1+\frac{\pi\left(\beta_{2}+\beta_{r}\right) N T_{s}}{3} \gamma_{T A S}\right)^{-(N-2)}\right\}\right]^{M}
\end{aligned}
$$


The lower bound outage probability at the relay node $R$ is defined as

$$
P_{\text {out }, \text { relay }}^{P-P N(\text { Lower })}[\gamma]=\operatorname{Pr}\left[\min \left(\gamma_{1, \text { relay }}^{P-P N}[k], \gamma_{2, \text { relay }}^{P-P N}[k]\right) \leq \gamma_{T A S}\right]
$$

Equation (37) is written in terms of CDF of $\gamma_{1, \text { relay }}^{P-P N}$ and $\gamma_{2, \text { relay }}^{P-P N}$ as,

$$
P_{\text {out }, \text { relay }}^{P-P N(\text { Lower })}=1-\left(1-F_{\gamma_{1}}^{P-P N}(\gamma)\right)\left(1-F_{\gamma_{2}}^{P-P N}(\gamma)\right)
$$

Since $F_{\gamma_{m}}^{P-P N}(\gamma)=P_{o u t, \gamma_{m}}^{P-P N}[\gamma], m=1,2$, substituting Equation (35) in Equation (38) the lower bound outage probability $P_{\text {out } \text {,elay }}^{P-P N(\text { Lower })}[\gamma]$ at relay node $R$ is determined as

$$
\begin{aligned}
& P_{\text {out }, \text { relay }}^{P-P N(\text { Lower })}[\gamma] \\
& =1-\exp \left(\begin{array}{l}
\left.\left[-\gamma_{T A S}\left\{\frac{1}{S N R}\left(1+\frac{\pi\left(\beta_{1}+\beta_{m}\right) N T_{s}}{3} \gamma_{T A S}\right)^{-(N-2)}\right\}\right]^{M}\right) \\
\left.-\left[\gamma_{T A S}\left\{\frac{1}{S N R}\left(1+\frac{\pi\left(\beta_{2}+\beta_{m}\right) N T_{s}}{3} \gamma_{T A S}\right)^{-(N-2)}\right\}\right]^{M}\right)
\end{array}\right)
\end{aligned}
$$

\subsection{Outage Analysis at Time Slot - II}

Let $P_{o u t, m}^{P-P N}[\gamma]$ be the outage probability at the link between the relay node $R$ and source node $S_{m}$ at $k^{\text {th }}$ subcarrier in time slot II. It is defined as $P_{o u t, m}^{P-P N}[\gamma]=\operatorname{Pr}\left[\gamma_{m, d e s t}^{P-P N}[k] \leq \gamma_{M R C}\right]$.

Substituting for $\gamma_{m, d e s t}^{P-P N}[k]$ from the Equation (25) it is written as

$$
P_{o u t, m}^{P-P N}[\gamma]=\operatorname{Pr}\left[\frac{\left(\sum_{b=1}^{M}\left|\tilde{g}_{m, b}[k]\right|^{2}\right)}{\left(\left(\frac{\pi\left(\beta_{r}+\beta_{m}\right) N T_{s}}{3}\right)\left\{\sum_{b=1}^{M} \sum_{q=0, q \neq k}^{N-1}\left|\tilde{g}_{m, b}[q]\right|^{2}\right\}+\frac{\sigma_{m}^{2}}{E_{r}}\right)} \leq \gamma_{M R C}\right], k=0,1, \ldots, N-1, m=1,2
$$

Equation (40) can be written as 


$$
P_{o u t, m}^{P-P N}[\gamma]=\operatorname{Pr}\left[\frac{X_{m}}{U_{m} Y_{m}+V_{m}} \leq \gamma_{M R C}\right], \quad m=1,2
$$

Let $\quad X_{m}=\left|\tilde{g}_{m, a_{m . k}}[k]\right|^{2}, \quad Y_{m}=\sum_{q=0, q \neq k}^{N-1}\left|\tilde{g}_{m, a_{m . k}}[q]\right|^{2}, \quad U_{m}=\frac{\pi\left(\beta_{m}+\beta_{r}\right) N T_{s}}{3} \quad$ and $V_{m}=\frac{\sigma_{r}^{2}}{E_{m}}=\frac{1}{S N R}$

Using the PDF of $X_{m}$ and $Y_{m}$ Equation (41) can be written as

$$
P_{o u t, m}^{P-P N}[\gamma]=\int_{0}^{\infty} \int_{0}^{\gamma\left(U_{m} y_{m}+V_{m}\right)} f_{X_{m}}\left(x_{m}\right) f_{Y_{m}}\left(y_{m}\right) d x_{m} d y_{m}
$$

Since $\tilde{g}_{m, b}[k]$ is circularly symmetric complex Gaussian random variable with zero mean and unit variance, the random variable $X_{m}$ is chi - square distributed with $2 M$ degrees of freedom and the random variable $Y_{m}$ is chi-square distributed with $2 M(N-1)$ degrees of freedom. Further evaluating the integrals the outage probability $P_{o u t, m}^{P-P N}[\gamma]$ at the source node $S_{m}$ during the time slot II is determined as $P_{\text {out }, m}^{P-P N}[\gamma]=1$

$$
-\exp \left(-\gamma_{M R C} / S N R\right)\left\{\begin{array}{c}
\left(1+\frac{\gamma_{M R C}}{S N R}\right)\left(1+\frac{\gamma_{M R C}\left(\pi\left(\beta_{r}+\beta_{m}\right) N T_{s}\right)}{3}\right)^{-2(N-1)-2} \\
+\frac{\gamma_{M R C}\left(\pi\left(\beta_{r}+\beta_{m}\right) N T_{s}\right)}{3}\left(2(N-1)\left(1+\frac{\gamma_{M R C}\left(\pi\left(\beta_{r}+\beta_{m}\right) N T_{s}\right)}{3}\right)^{-2(N-1)-1}\right) \\
m=1,2
\end{array}\right\}
$$

\subsection{End - To - End Outage Analysis}

The overall upper bound end - to - end outage probability is expressed as

$$
P_{\text {out }, E-E}^{P-P N(\text { Upper })}[\gamma]=P_{\text {out }, \text { relay }}^{P-P N(\text { Upper })}[\gamma]+\left[1-P_{\text {out }, \text { relay }}^{P-P N(\text { Upper })}[\gamma]\right]\left[P_{\text {out }, m}^{P-P N}[\gamma]\right]
$$


By substituting Equation (36) and Equation (43) in Equation (44) the closed form expression of the overall upper bound end - to - end outage probability is obtained as $P_{\text {out }, E-E}^{P-P N(\text { Upper })}[\gamma]=5-\left(3+\exp \left[-\gamma_{\text {TAS }}\left\{\frac{1}{S N R}\left(1+\frac{\pi\left(\beta_{1}+\beta_{r}\right) \beta_{1} N T_{s}}{3} \gamma_{T A S}\right)^{-(N-2)}\right\}\right]^{M}\right)$
$\left.+\exp \left[-\gamma_{T A S}\left\{\frac{1}{S N R}\left(1+\frac{\pi\left(\beta_{2}+\beta_{r}\right) N T_{s}}{3} \gamma_{T A S}\right)^{-(N-2)}\right\}\right]^{M}\right]$
$\times \exp \left(-\gamma_{M R C} / S N R\right)\left\{\begin{array}{l}\left(1+\frac{\gamma_{M R C}}{S N R}\right)\left(1+\frac{\gamma_{M R C}\left(\pi\left(\beta_{r}+\beta_{m}\right) N T_{s}\right)}{3}\right)^{-2(N-1)-2} \\ \left(\pi\left(\beta_{r}+\beta_{m}\right) N T_{s}\right) \\ 3\end{array}\right)$

The overall lower bound end - to - end outage probability is expressed as

$$
P_{\text {out }, E-E}^{P N(\text { Lower })}[\gamma]=P_{\text {out }}^{P-P N(\text { Lower })}[\gamma]+\left[1-P_{\text {out }}^{P-P N(\text { Lower })}[\gamma]\right]\left[P_{\text {out }, m}^{P-P N}[\gamma]\right]
$$

Similarly, by substituting Equation (39) and Equation (43) in Equation (46) the closed form expression for the overall lower bound end - to - end outage probability is obtained as 


$$
\begin{aligned}
& P_{\text {out }, E-E}^{P N(\text { Lower })}[\gamma]=1-\exp \left(\begin{array}{l}
{\left[-\gamma_{T A S}\left\{\frac{1}{S N R}\left(1+\frac{\pi\left(\beta_{1}+\beta_{m}\right) N T_{s}}{3} \gamma_{T A S}\right)^{-(N-2)}\right\}\right]^{M}} \\
-\left[\gamma_{T A S}\left\{\frac{1}{S N R}\left(1+\frac{\pi\left(\beta_{2}+\beta_{m}\right) N T_{s}}{3} \gamma_{T A S}\right)^{-(N-2)}\right\}\right]^{M}
\end{array}\right) \\
& \times \exp \left(-\gamma_{M R C} / S N R\right)\left\{\begin{array}{l}
\left(1+\frac{\gamma_{M R C}}{S N R}\right)\left(1+\frac{\gamma_{M R C}\left(\pi\left(\beta_{r}+\beta_{m}\right) N T_{s}\right)}{3}\right)^{-2(N-1)-2} \\
+\frac{\gamma_{M R C}\left(\pi\left(\beta_{r}+\beta_{m}\right) N T_{s}\right)}{3} \\
\left(2(N-1)\left(1+\frac{\gamma_{M R C}\left(\pi\left(\beta_{r}+\beta_{m}\right) N T_{s}\right)}{3}\right)^{-2(N-1)-1}\right.
\end{array}\right\}
\end{aligned}
$$

\section{RESULTS AND DISCUSSION}

In this section, the outage performance of the proposed transceiver in the presence of phase noise is analyzed. The parameter used in the numerical results is given in Table 1.

Table 1 Numerical parameters of the bidirectional relay network in the presence phase noise with multiple antennas

\begin{tabular}{|l|l|l|}
\hline Symbol & \multicolumn{1}{|c|}{ Parameter } & \multicolumn{1}{|c|}{ Value } \\
\hline$N$ & Number of Subcarriers & 64 \\
\hline$T_{s}$ & OFDM Symbol duration & $50 \mathrm{~ns}$ \\
\hline$\beta$ & Phase noise Bandwidth & $100,500,1000(\mathrm{~Hz})$ \\
\hline$R_{d}$ & Data rate & $1 \mathrm{bits} / \mathrm{s} / \mathrm{Hz}, 0.5 \mathrm{bits} / \mathrm{s} / \mathrm{Hz}$ \\
\hline $\mathrm{M}$ & $\begin{array}{l}\text { Number of Transmit and Receive antenna at } \\
\text { source node } S_{m}\end{array}$ & 2,4 \\
\hline
\end{tabular}




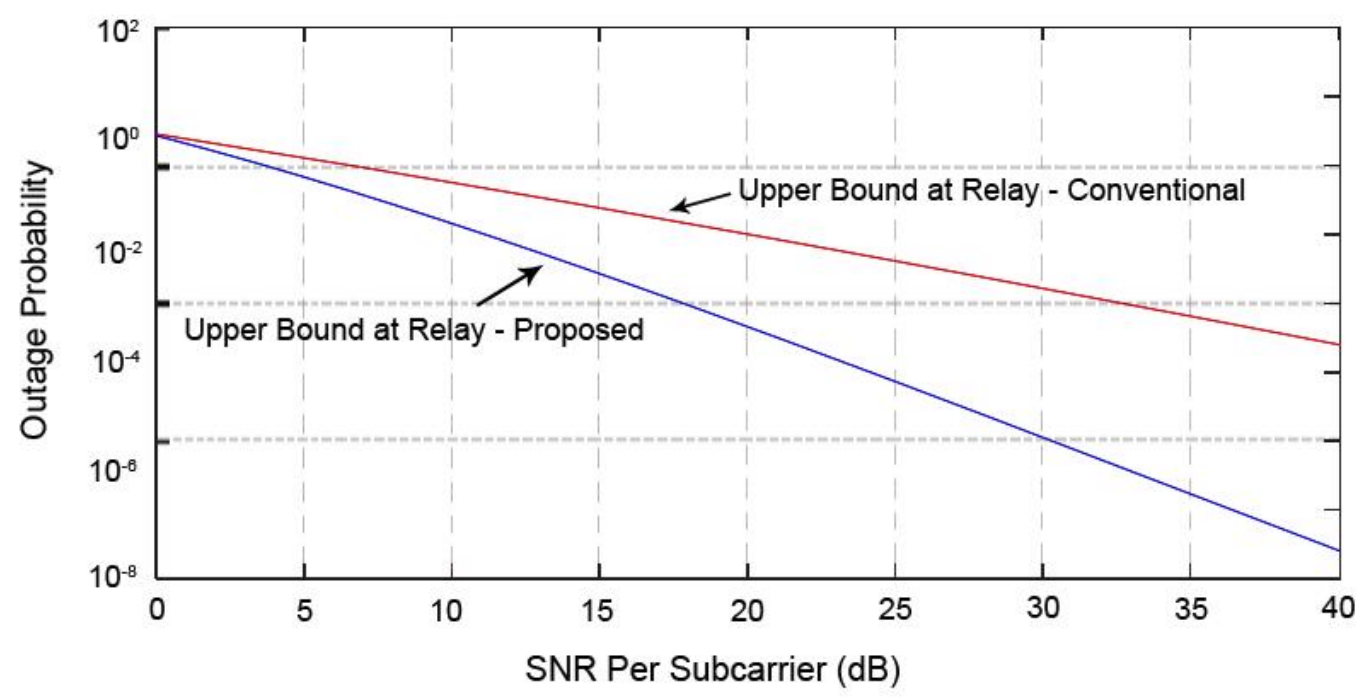

Figure 2 Outage performance of OFDM based bidirectional relay network at relay node

Figure 2 shows the upper bound outage performance comparison of proposed transceiver structure with the conventional transceiver structure at relay node in OFDM based bidirectional relay network in the absence of phase noise. It is observed in high SNR region $15 \mathrm{~dB}$ improvements in the proposed transceiver structure when compared to the conventional structure.

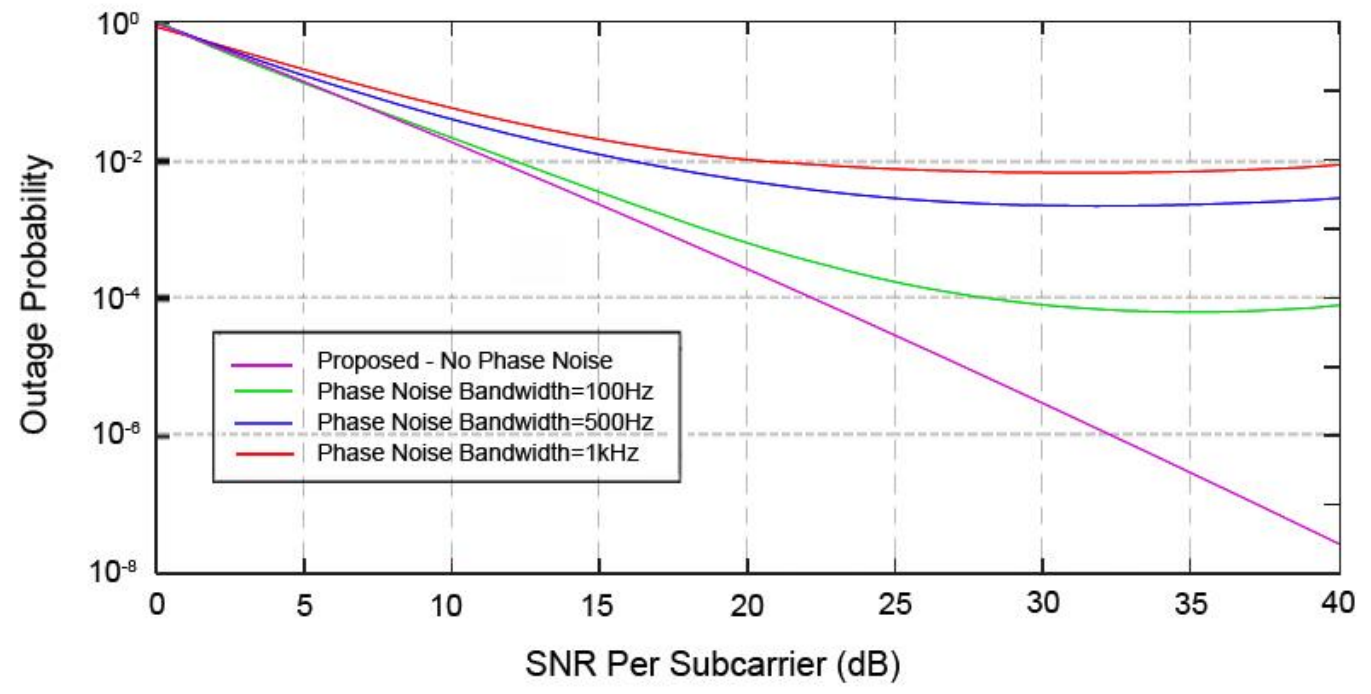

Figure 3 Outage performance at the relay node with phase noise

Figure 3 shows the outage performance of the proposed transceiver with phase noise variations at the relay node $R$. It is observed that as phase noise bandwidth increases from $500 \mathrm{~Hz}$ to $1 \mathrm{kHz}$ the outage probability at the relay node also increases. 


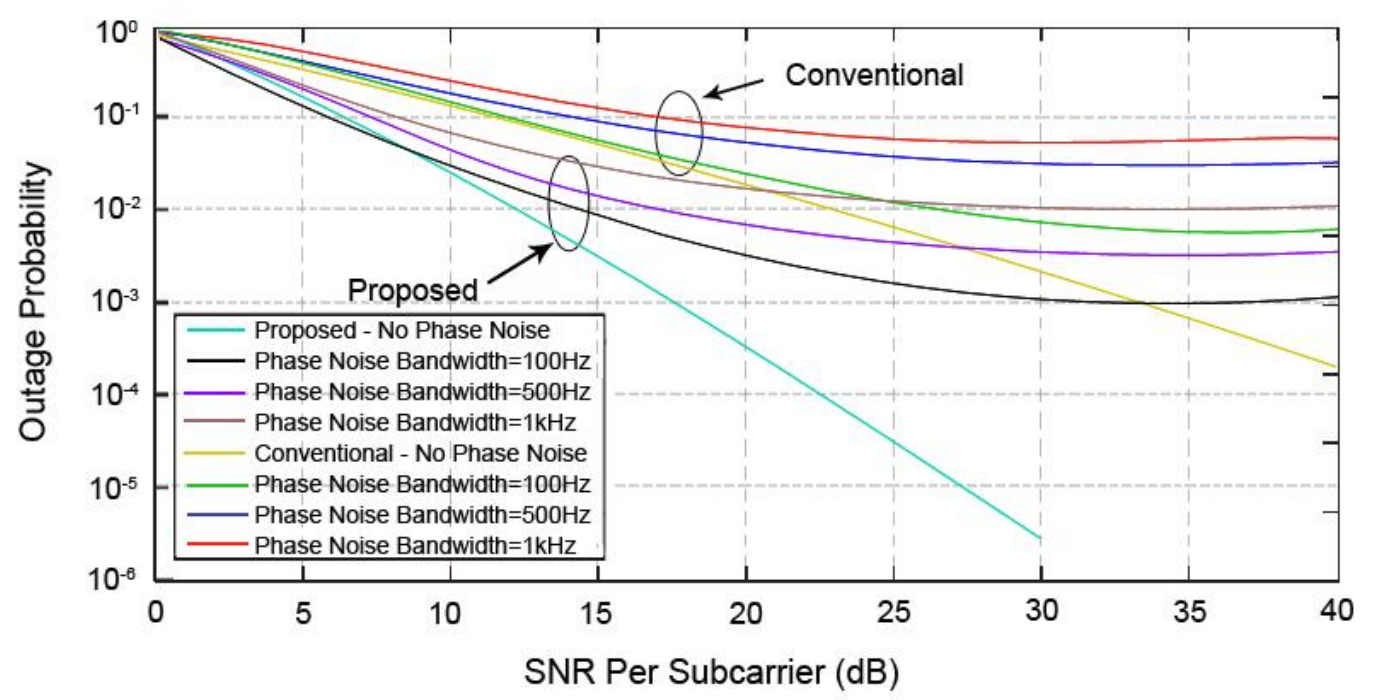

Figure 4 End-to-End outage performance comparisons between conventional method and proposed method

Figure 4 shows the comparison of the end to end upper bound outage performance of the proposed transceiver structure with the conventional transceiver structure. In the absence of phase noise both conventional and proposed method performs well. Approximately $15 \mathrm{~dB}$ improvement in the proposed method compared to the conventional in the absence of phase noise. It is observed that when the phase noise bandwidth increases from $100 \mathrm{~Hz}$ to $1000 \mathrm{~Hz}$, the outage probability also increases from and further increase in the phase noise bandwidth, the system reaches deep fade.

\section{CONCLUSION}

In this paper, PLNC and OFDM based bidirectional relay network is modeled with phase noise. The analytical expressions for outage probability are derived at the relay node in time slot I, destination nodes in time slot II. Based on the performance analysis of outage, novel transceiver architecture for bidirectional relay network is proposed. In the proposed architecture, multiple antennas are at source nodes. TAS is performed at the source nodes such that PLNC can be carried out at the relay node without any complexity. It provides selection diversity in time slot I. MRC is carried out in time slot II to obtain spatial diversity. The proposed network is also modeled with phase noise. Further, analytical expressions are also derived for analyzing the outage performances of the proposed network in the presence of phase noise. Based on the order of selection diversity and spatial diversity, 
the proposed transceiver architecture provides improved outage performances than the conventional bidirectional network.

\section{Conflict of Interest Statement:}

On behalf of all authors, the corresponding author states that there is no conflict of interest.

\section{REFERENCES}

1. Zhang, Y., Member, S., \& Liu, H. (2007). MIMO-OFDM Systems in the Presence of Phase Noise and Doubly Selective Fading, 56(4), 2277-2285.

2. Yang, W., Cai, Y., Hu, J., \& Yang, W. (2010). Channel Estimation for Two-Way Relay OFDM Networks, 2010. doi:10.1155/2010/186182

3. Sun, Y., Yang, Y., Si, P., Yang, R., \& Zhang, Y. (2016). Novel self-interference suppression schemes based on Dempster-Shafer theory with network coding in twoway full-duplex MIMO relay. EURASIP Journal on Wireless Communications and Networking, 1-16. doi:10.1186/s13638-016-0597-1

4. Sivabalan, V., Malaiyappan, N. M., \& Thiruvengadam, S. J. (2014). Full Duplex Relay Based Cognitive Radio System with Physical Layer Network Coding, (November 2015). doi:10.1007/s11277-014-2075-6

5. Liao, K., Zhao, S., Long, Y., Huang, G., \& Tang, D. (2019). Distributed Beamforming Design for Nonregenerative Two-Way Relay Networks with Simultaneous Wireless Information and Power Transfer, 2019.

6. Pan, L., Li, Z., Wang, Z., \& Zhang, F. (2019). Joint Relay Selection and Power Allocation for the Physical Layer Security of Two-Way Cooperative Relaying Networks, 2019.

7. Narmanlioglu, O., Turan, B., Coleri, S., \& Uysal, M. (2018). Cooperative MIMOOFDM based inter-vehicular visible light communication using brake lights. Computer Communications, 120(December 2016), 138-146. doi:10.1016/j.comcom.2018.02.003

8. Engineering, C. (n.d.). Optimal Relay Power Allocation for Amplify-and-Forward Relay Networks with Non-linear Power Amplifiers.

9. Liu, K., Zhu, Q., \& Wang, Y. (2018). Outage Analysis and Power Allocation Optimization for Multiple Energy-Harvesting Relay System Using SWIPT, 2018.

10. Ravindran Unnithan Jalaja, R., Periakarupan Gurusamy Sivabalan, V., \& Sundarrajan Jayaraman, T. (2019). Energy efficient bidirectional relay network with spatial 
modulation. International Journal of Communication Systems, (May), 1-11. doi:10.1002/dac.4187

11. Rajakumar, G., \& Roobert, A. A. (2018). Design of Low Power VLSI Architecture of Line Coding Schemes. Wireless Personal Communications. doi:10.1007/s11277-018$5286-4$

12. Li, J., Bao, J., Luan, S., Jiang, B., \& Liu, C. (2018). Power Optimized Single Relay Selection with an Improved Link-Adaptive-Regenerative Protocol, 2018. 
Figures

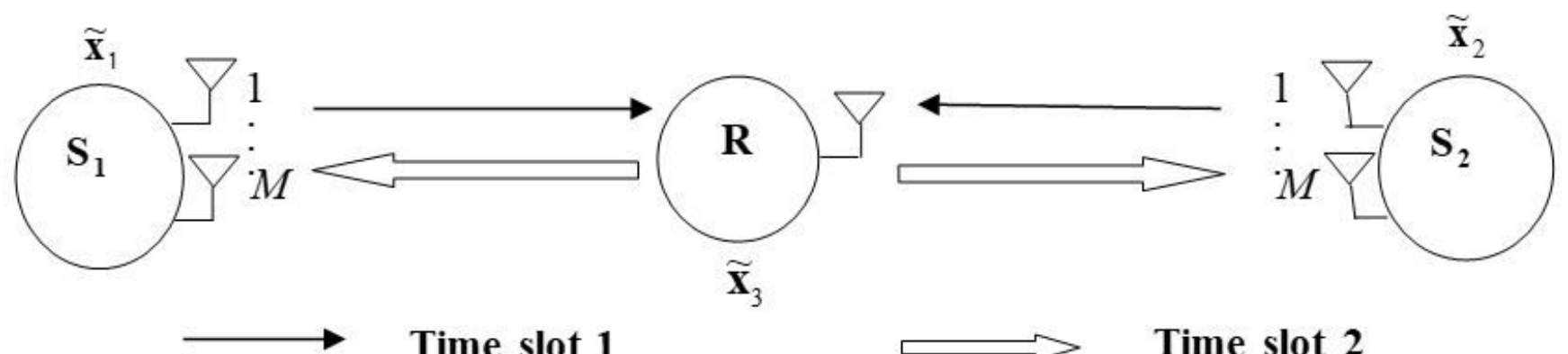

Figure 1

System model



Figure 2

Outage performance of OFDM based bidirectional relay network at relay node 


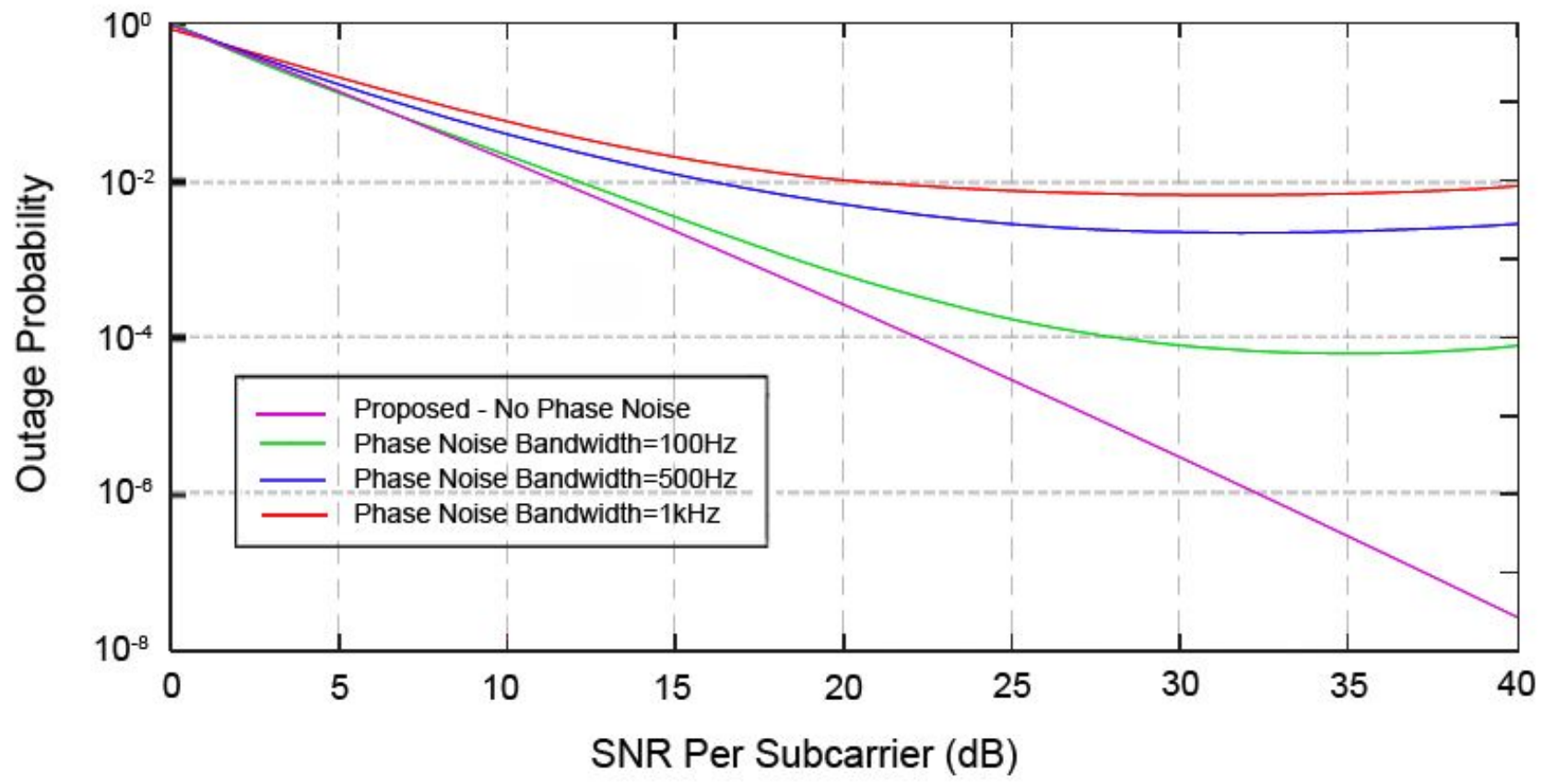

Figure 3

Outage performance at the relay node with phase noise



Figure 4

End-to-End outage performance comparisons between conventional method and proposed method 